\title{
A New Bayesian Network Method for Computing Bottom Event's Structural Importance Degree using Jointree
}

\author{
Wang Yao and Sun Qin \\ School of Aeronautics, Northwestern Polytechnical University, Xi'an, 710072 PR \\ China) \\ wangyaorose@126.com,sunqing@nwpu.edu.cn
}

\begin{abstract}
$T$ Bayesian network methodology taking place of fault tree analysis used for reliability assessment has gotten lots of attention in recent years. On basis of the current Bayesian network method used for calculating structural importance degree, a new Bayesian network method is raised. This new method can avoid repeat modification of the parameters in the conditional probability tables, and hence the intermediate results computed during inference process can be shared to decrease calculating complexity. The new method is proved to be correct in a mathematic way and a corresponding algorithm named SID_Jointree for realizing this new method is designed, which guarantees the new method can be realized in computer. Finally, the correctness and efficiency of the new method is validated by using two fault tree cases.
\end{abstract}

Keywords: Bayesian network; fault tree; structural importance degree; reliability assessment; inference

\section{Introduction}

As a model used for reliability assessment, Bayesian network mapped from a fault tree can achieve more useful results than fault tree analysis (FTA). Studies show that, all the results calculated through FTA can be computed through Bayesian network and the converse is not true. For example, if the top event occurs, one can calculate the posterior probability of the bottom event using Bayesian network, which can't be calculated using FTA.

The methods for evaluating system using a fault tree's equivalent Bayesian network have gotten lots of attention home and abroad. In special, many researchers focus on the methods for solving reliability indexes, such as minimal cut sets, minimal path cuts, structural importance degree, and probability importance degree. Among these researchers, Luigi Portinale [1,2] and Nima Khakzad [3] studied how to solve the minimal cut sets and how to calculate reliability degree in a mathematical way; Weidl [5] studied how to calculate the reliability degree and some other traditional reliability indexes using the existent Bayesian network toolbox. Zhou Zhongbao [6] raised a new method for computing the structural importance degree, which represents the new state-of-the-art in solving such a traditional reliability index using Bayesian network. However, the new method is complicated: for each bottom event, one needs to inference in the equivalent Bayesian network once. That's to say, for a system with $n$ bottom events, one has to inference $n$ times in the Bayesian network. As $n$ increases, the efficiency of the method decreases. On the basis of researches stated above, this paper presents a new method for calculating all the bottom events' structural importance degree using a Jointree extracted from the fault tree's equivalent Bayesian network, as well as the corresponding algorithm SID_Jointree. Jointree is a kind of structural that the intermediate results can be stored in the edges of itself during inference, so through Jointree 
one can reduce the inference complexity by sharing the stored results [7-8]. In fact, through an inward and outward inference in the Jointree (both inward and outward inference is equivalent to inference once using variable elimination algorithm in a Bayesian network, separately [8]), one can store all the intermediate results needed for calculating each node's posterior probability, which is the key kind of probability for calculating each node's structural importance degree. However, according to Zhou's method, one has to change other nodes' CPTs before calculating one bottom event's structural importance degree in a Bayesian network. Once any CPT changed in a Bayesian network, the intermediate results stored in the edges of Bayesian network's corresponding Jointree become invalid and cannot be shared for calculating another bottom event's structural importance degree. That means one has to calculate each bottom event's structural importance degree one by one. On the contrary, the method raised in this paper avoids changing any node's CPT during inference. Thus, the intermediate results stored in the edges of the Jointree are still valid for calculating any other event's structural importance degree. In addition, the new method is proved correct in a mathematical way. Finally, such a method is exemplified with two fault cases.

\section{Bayesian Network}

\subsection{Definition of Bayesian Network}

Bayesian network is a directed acyclic graph, consisting of nodes denoted by variables and directed edges that point from father nodes to children nodes, each edge denoted by one-line arrows [7].

Every node in Bayesian network has a conditional probability table (CTP). To be specific, the CPT of node $Y_{i}$ can be represented with $\operatorname{Pr}\left(Y_{i} \mid \pi\left(Y_{i}\right)\right)$, in which $\pi\left(Y_{i}\right)$ stands for the set of node $Y_{i}$ 's fathers. Variable $Y_{i}$ and its fathers $\pi\left(Y_{i}\right)$ make up a family. Intuitively, every CPT stores its family's possible states, as well as the states' corresponding probabilities. A simple Bayesian network is shown in Fig.1 (b).

For a Bayesian network, in which all the variables make up set $N=\left\{Y_{1}, Y_{2}, \cdots, Y_{n}\right\}$, joint probability distribution of the network can be computed by multiplying all the nodes' CPTs: $\operatorname{Pr}\left(Y_{1}, Y_{2}, \cdots, Y_{n}\right)=\prod_{i=1}^{n} \operatorname{Pr}\left(Y_{i} \mid \pi\left(Y_{i}\right)\right)[8]$.

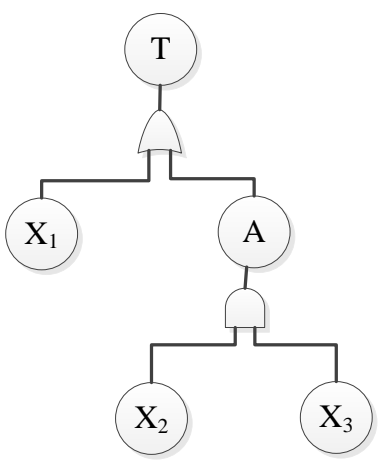

(a) 


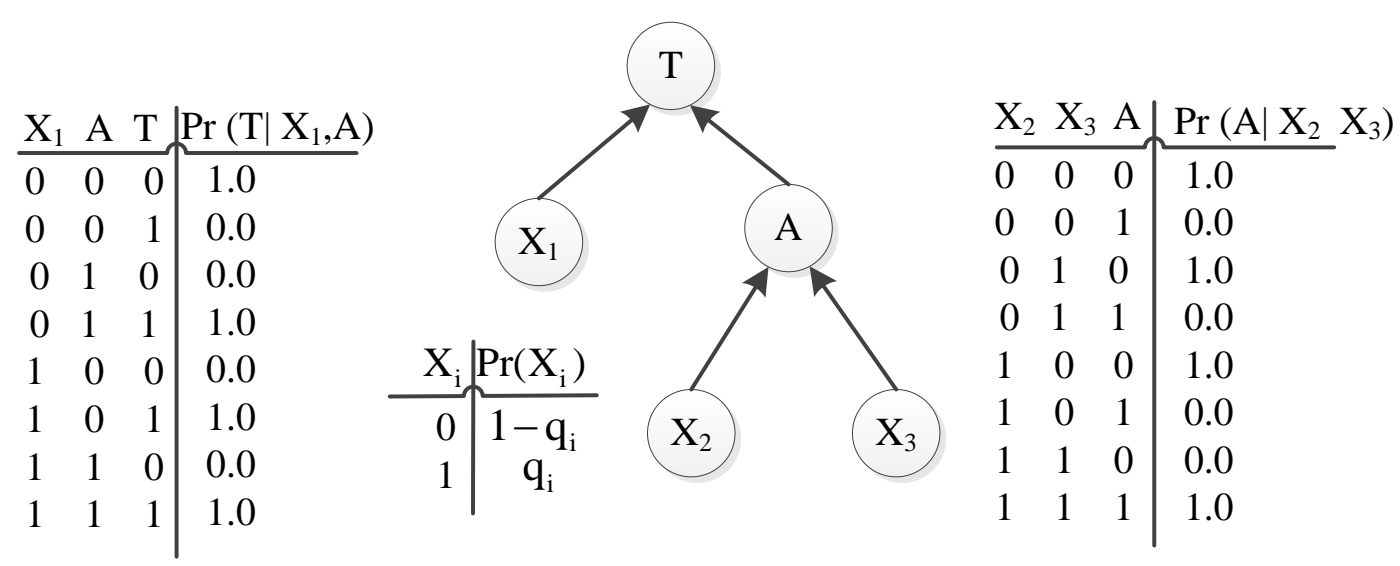

(b)

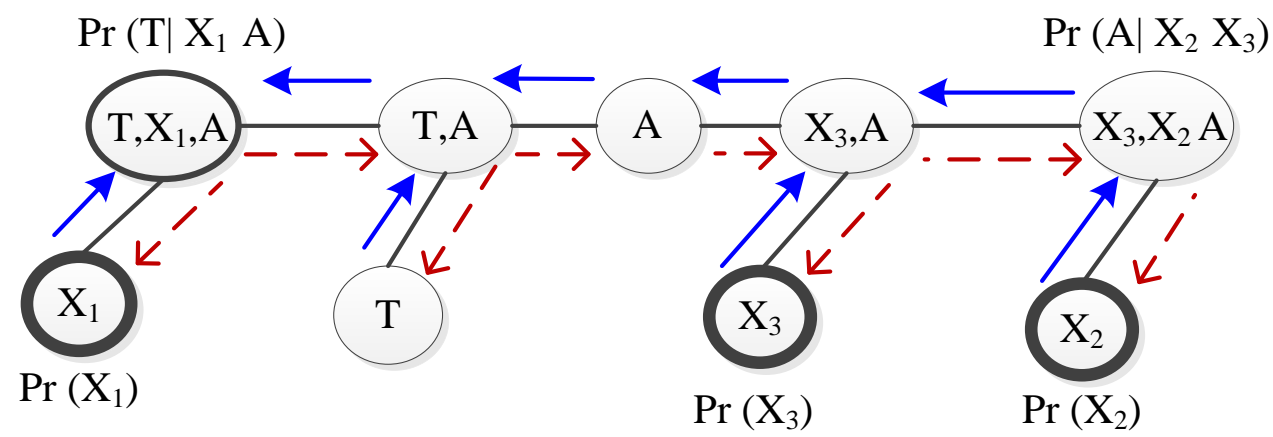

(c)

Figure 1. A Simple System Described in Different Topologies

(a) Fault Tree; (b) The equivalent Bayesian network; (c) A Jointree extracted from the Bayesian network with each CPT assigned to their family clusters, taking the cluster $\left\{T, X_{1}, A\right\}$ as root, solid arrows indicate the direction of inward calculation and dotted arrows indicate the direction of outward calculation. After an inward and outward calculation, intermediate results can be stored in the edges or clusters. And If intermediate results can be shared, $\operatorname{Pr}\left(X_{i} \mid \boldsymbol{E}=\boldsymbol{e}\right)$ can be read from cluster $\left\{X_{i}\right\}$ directly.

\subsection{Inference in Bayesian Networks}

All the inference problems in Bayesian network can be boiled down to solving the probability distribution of $\operatorname{Pr}(\boldsymbol{Q}, \boldsymbol{E}=\boldsymbol{e})$, in which $\boldsymbol{Q}$ stands for the set of query variables that people are interested in and $\boldsymbol{E}$ stands for the set of evidence variables that people have already known its values $\boldsymbol{e}$.

Mainstream, variable elimination algorithm (VE) and Jointree algorithm are two main exact inference algorithms in Bayesian network.

Jointree algorithm starts with a jointree extracted from Bayesian network (see Fig. 1(c)).For a Bayesian network, it has many different Jointrees according to different construction algorithms. In this paper, one kind of Jointree named binary Jointree is adopted by using the construction algorithm stated in the literature $[9,10]$. Nodes of jointree are called clusters and each cluster contains one or several variables. As stated before, if a cluster 
consists of a variable with all its fathers, the cluster is called family cluster. After a jointree is extracted, assign every CPT to its family cluster. Then a jointree can be viewed as a special structure that one can store intermediate results in the edges or clusters during inference process and these intermediate results can be shared when solving another different Bayesian network inference problem. Such a data-shared mechanism can avoid more calculation in solving another inference problem. Because of these shared intermediate data, one can compute the probability distribution $\operatorname{Pr}\left(Y_{i}, \boldsymbol{E}=\boldsymbol{e}\right)$ of each node $Y_{i}$ by just performing Jointree algorithm once. Here $Y_{i}$ can represent any node in the network. But when any CPT is changed, the intermediate results stored in the jointree become invalid. One has to perform the Jointree algorithm again once any CPT has been changed.

VE algorithm doesn't store any intermediate results in inference process. Therefore, there is no shared data when solving another inference problem. One can only compute the probability distribution $\operatorname{Pr}\left(Y_{i}, \boldsymbol{E}=\boldsymbol{e}\right)$ of someone node $Y_{i}$ by VE algorithm. Here, $Y_{i}$ stands only some node in the network, not any node.

In fact, Jointree algorithm contains two computation parts: inward calculation and outward calculation. Each kind of calculation is equivalent to performing VE algorithm once except that intermediate results are store during performing inward and outward calculation while VE algorithm stores nothing. Taking Fig.1(c) for an instance, set evidence $T=1$. Using Jointree algorithm, one can extract the results $\operatorname{Pr}\left(X_{i}, T=1\right), i=1,2,3$ from the cluster $\left\{X_{i}\right\}$ after an inward and outward calculation. While one needs to perform VE algorithm three times in order to calculate $\operatorname{Pr}\left(X_{i}, T=1\right), i=1,2,3$.

\subsection{Mapping Fault Trees into Bayesian Networks}

Denote a fault tree using $\overline{F T}$ and denote its equivalent Bayesian network using $\overline{B N}$. On the basis of references [1-3], the steps for mapping $\overline{F T}$ into $\overline{B N}$ are given as below.

1. For every bottom event in $\overline{F T}$, build only one node in $\overline{B N}$ to correspond to. Then draw arrows that point from nodes which are correspondent with bottom events to nodes which are correspondent with intermediate/top events. Specifically, in $\overline{B N}$, variable $T$ denotes the node that corresponds to top event, and $T=0 / 1$ holds; $X_{i}$ denotes the node that corresponds to bottom event, and $X_{i}=0 / 1$ holds; $A_{j}$ denotes the node that corresponds to intermediate event, and $A_{j}=0 / 1$ holds. In the following, " 1 " represents the fault state and " 0 " represents the normal/workable state.

2. Determine every root node' $\mathrm{CPT}$, denoted by $\operatorname{Pr}\left(X_{i}\right)$. Root nodes are mapped from bottom events, therefore $\operatorname{Pr}\left(X_{i}\right)$ is decided by each bottom event's failure probability qi. To be specific, $\operatorname{Pr}\left(X_{i}=1\right)=q_{i}$ and $\operatorname{Pr}\left(X_{i}=0\right)=1-q_{i}$ holds.

3. Determine non-root nodes' CPTs, denoted by $\operatorname{Pr}(T \mid \pi(T))$ or $\operatorname{Pr}\left(A_{j} \mid \pi\left(A_{j}\right)\right)$. These non-root nodes are mapped from intermediate/top events. These events are caused by bottom events in some kind of deterministic logic relationship, such as "AND" and "VOTE". The CPT of non-root node must be given corresponding to such a deterministic relationship. The detailed method for generating non-root node's CPT is discussed in $[1,6]$.

According to the procedures above, the equivalent Bayesian network of Figure 1 (a) is given in Figure 1 (b). 


\section{Methods for Solving Structural Importance Degree}

\subsection{FTA Method}

The state of top event is a structural function (or called state function), which varies with every bottom event's state, denoted by $\phi(X)=\phi\left(X_{1}, X_{2}, \cdots, X_{n}\right)$. Both $\phi(X)$ and $X_{\mathrm{i}}$ take the value 1 or 0 . Using FTA, the structural importance degree of bottom event $X_{\mathrm{i}}$ is computed by Eq. (1) [11] based on $\overline{F T}$.

$$
\begin{gathered}
I_{\phi(i)}=\frac{1}{2^{n-1}} \sum_{k_{1}, k_{2} \cdots k_{i-1}, k_{i+1}, \cdots k_{n}=0}^{1}\left[\phi\left(X_{i}=1, X_{1}=k_{1}, X_{2}=k_{2} \cdots, X_{i-1}=k_{i-1}, X_{i+1}=k_{i+1}, \cdots, X_{n}=k_{n}\right)-\right. \\
\left.\phi\left(X_{i}=0, X_{1}=k_{1}, X_{2}=k_{2} \cdots, X_{i-1}=k_{i-1}, X_{i+1}=k_{i+1}, \cdots, X_{n}=k_{n}\right)\right]
\end{gathered}
$$

In Eq. (1), the summation symbol means that all the variables inside the bracket should traverse the value 0 and 1 except the bottom event node $X_{i}$.

\subsection{Bayesian Network Method}

3.2.1. Existent Bayesian Network Method: Eq. (1) provides an exact way to solve bottom event $\mathrm{X}_{\mathrm{i}}$ 's structural importance degree. In essence, it is an exhaust method that one should list a system's every possible state. For example, if a system has $n$ bottom events, the system will have $\exp (\mathrm{n})$ states and one has to list all these states to computing the index. As n increase, listing all the possible states is a hardly work. In contrast, the Bayesian network method proposed by Zhou [6] avoids such an exhaust list, which makes exact computation feasible in a larger system.

According to Zhou's method, node $\mathrm{X}_{\mathrm{i}}$ 's structural importance degree can be computed using Eq. (2) in $\overline{B N}$ :

$$
I_{\phi(i)}=\operatorname{Pr}\left(T=1 \mid X_{i}=1\right)-\operatorname{Pr}\left(T=1 \mid X_{i}=0\right)
$$

$X_{i}$ is the node whose structural importance degree to be calculated. However, for Eq. (2) there are the following calculation conditions. Before using Eq.(2), the non-root nodes' CPTs should remain unchanged just as their original CPTs generated in the first beginning using the mapping method in Section 2.3, as well as node $X_{i}$ 's CPT; Meanwhile, all the root nodes' CPTs except $X_{\mathrm{i}}$ 's should be changed to a uniform distribution as follows:

$$
\operatorname{Pr}\left(X_{j}=0\right)=1 / 2, \operatorname{Pr}\left(X_{j}=1\right)=1 / 2, j \neq i \text {. }
$$

According to Eq. (2) and its calculation conditions, when solving $X_{i}$ 's structural importance degree, the rest root nodes' CPTs have to be changed. Therefore, intermediate results cannot be shared even though Jointree algorithm is adopted (Obviously, the intermediate results stored in Jointree become invalid when solving another bottom event because some CPTs have to be changed again). One has to solve each bottom event's structural importance degree one by one.

Above all, using the existent Bayesian network method to solve a system with $\mathrm{n}$ bottom events, Eq. (2) has to be computed $n$ times.

3.2.2. A New Bayesian Network Method: Here a new method is raised and will be proved in a mathematical way. The new Bayesian network method is expressed with Eq. (3) as below:

$$
I_{\phi(i)}=2\left[\operatorname{Pr}\left(T=1, X_{i}=1\right)-\operatorname{Pr}\left(T=1, X_{i}=0\right)\right]
$$

Before calculating Eq. (3), set every root node's CPT to a uniform distribution as follows:

$$
\operatorname{Pr}\left(X_{j}=0\right)=1 / 2, \operatorname{Pr}\left(X_{j}=1\right)=1 / 2 \text {. }
$$


Comparing the new method with Eq. (2), a major difference lies in that all the CPTs are set once and none of them will be changed again using the new method while using Eq. (2), some root nodes' CPTs have to be changed again when solving a different bottom event's structural importance degree. No changing CPTs any more means that intermediate results will be still useful and can be shared when solving another different bottom event's structural importance degree using Jointree algorithm (As stated in Section 2.2, Jointree algorithm can store intermediate results, and if the CPTs are never been changed, these intermediate data will valid forever ). Therefore, one can compute every bottom event's structural importance degree just performing Jointree algorithm once. In what follows, Eq. (3) is proved in a mathematical way.

\section{Proof:}

As structural importance degree is irrespective of the failure probability of each bottom event of $\overline{F T}$, set each bottom event's failure probability equals to 0.5. Obviously, for each root node $\mathrm{X}_{\mathrm{i}}$ in $\overline{B N}$, its CPT is as below:

$$
\operatorname{Pr}\left(X_{j}=0\right)=1 / 2, \operatorname{Pr}\left(X_{j}=1\right)=1 / 2 .
$$

As $X_{1}, X_{2}, \cdots, X_{n-1}$ and $X_{n}$ are independent of each other, Eq. (4) and Eq. (5) hold.

$$
\begin{aligned}
& \operatorname{Pr}\left(X_{1}, X_{2}, \cdots, X_{i-1}, X_{i+1}, \cdots, X_{n}\right)=1 / 2^{n-1} \\
& \operatorname{Pr}\left(X_{1}, X_{2}, \cdots, X_{n}\right)=1 / 2^{n}
\end{aligned}
$$

Combining Bayer Theorem, Eq. (6) and Eq. (7) can be derived from Eq. (4) and Eq. (5).

$$
\begin{aligned}
& \operatorname{Pr}\left(T=1 \mid X_{i}=1, X_{1}=k_{1}, X_{2}=k_{2}, \cdots, X_{i-1}=k_{i-1}, X_{i+1}=k_{i+1}, \cdots, X_{n}=k_{n}\right) \\
= & \operatorname{Pr}\left(T=1, X_{i}=1, X_{1}=k_{1}, X_{2}=k_{2}, \cdots, X_{i-1}=k_{i-1}, X_{i+1}=k_{i+1}, \cdots, X_{n}=k_{n}\right) \cdot 2^{n} \\
& \operatorname{Pr}\left(T=1 \mid X_{i}=0, X_{1}=k_{1}, X_{2}=k_{2}, \cdots, X_{i-1}=k_{i-1}, X_{i+1}=k_{i+1}, \cdots, X_{n}=k_{n}\right) \\
= & \operatorname{Pr}\left(T=1, X_{i}=0, X_{1}=k_{1}, X_{2}=k_{2}, \cdots, X_{i-1}=k_{i-1}, X_{i+1}=k_{i+1}, \cdots, X_{n}=k_{n}\right) \cdot 2^{n}
\end{aligned}
$$

In $\overline{F T}$, sentence $\phi\left(X_{1}=k_{1}, X_{2}=k_{2}, \cdots, X_{i-1}=k_{i-1}, X_{i}=k_{i}, X_{i+1}=k_{i+1}, \cdots X_{n}=k_{n}\right)=0$ means that top event certainly won't happen when all the bottom events take the state $k_{1}, k_{2}, \cdots, k_{i-1}, k_{i}, k_{i+1}, \cdots, k_{n}$ listed in the Parentheses. In $\overline{B N}$, such a sentence means that top event $\mathrm{T}$ takes state 1 with a probability of 0.0. Analogously, in $\overline{F T}$ sentence $\phi\left(X_{1}=k_{1}, X_{2}=k_{2}, \cdots, X_{i-1}=k_{i-1}, X_{i}=k_{i}, X_{i+1}=k_{i+1}, \cdots X_{n}=k_{n}\right)=1$ means that the top event curtains to occur when all the bottom events take the state $k_{1}, k_{2}, \cdots, k_{i-1}, k_{i}, k_{i+1}, \cdots, k_{n}$ listed in the Parentheses. In $\overline{B N}$, such a sentence means that top event $\mathrm{T}$ takes state 1 with a probability of 1.0 .

According to the illustration above, Eq. (8) holds.

$$
\begin{aligned}
& \phi\left(X_{1}=k_{1}, X_{2}=k_{2}, \cdots, X_{i-1}=k_{i-1}, \cdots, X_{i+1}=k_{i+1}, \cdots, X_{n}=k_{n}\right) \\
= & \operatorname{Pr}\left(T=1 \mid X_{1}=k_{1}, X_{2}=k_{2}, \cdots, X_{i-1}=k_{i-1}, \cdots, X_{i+1}=k_{i+1}, \cdots, X_{n}=k_{n}\right)
\end{aligned}
$$

Take Eq. (6), Eq. (7) and Eq. (8) into Eq. (1), Eq. (9) can be achieved.

$$
\begin{aligned}
& I_{\phi(i)}=2 \sum_{k_{1}, \cdots k_{i-1}, k_{i+1}, \cdots k_{n}=0}^{1} \operatorname{Pr}\left(T=1, X_{i}=1, X_{1}=k_{1}, \cdots, X_{i-1}=k_{i-1}, X_{i+1}=k_{i+1}, \cdots, X_{n}=k_{n}\right)- \\
& \quad 2 \sum_{k_{1}, \cdots k_{i-1}, k_{i+1}, \cdots k_{n}=0}^{1} \operatorname{Pr}\left(T=1, X_{i}=0, X_{1}=k_{1}, \cdots, X_{i-1}=k_{i-1}, X_{i+1}=k_{i+1}, \cdots, X_{n}=k_{n}\right) \\
& =2\left[\operatorname{Pr}\left(T=1, X_{i}=1\right)-\operatorname{Pr}\left(T=1, X_{i}=0\right)\right]
\end{aligned}
$$

\section{Proof ends.}


Above all, for a system with $\mathrm{n}$ bottom events, one can compute all bottom events' structural importance degree with two times' inference using the new method: an inward computation and an outward computation.

3.2.3. The Corresponding New Algorithm SID_Jointree: Eq. (3) is a new mathematical method for computing each bottom event's structural importance degree. As a complement to the new method, the corresponding new algorithm SID_Jointree is designed. In fact, two times' inference (an inward and outward) is performed after calling SID_Jointree, which results in every bottom event's structural importance degree.

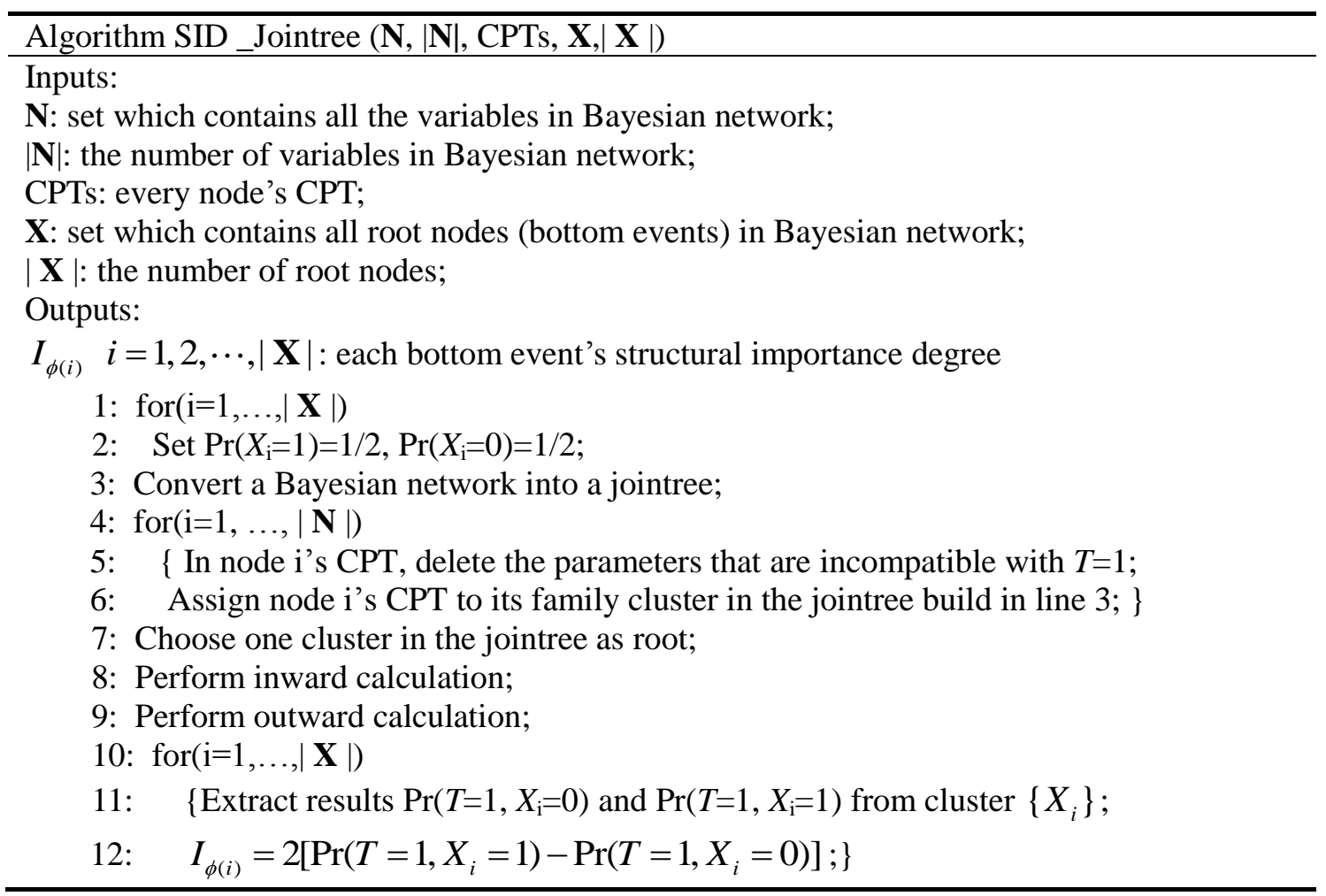

\section{Case Studies}

Two cases are discussed using the old and new Bayesian network method, separately. And the results are compared. The efficiency and feasibility of the new Bayesian network method is the main concern in this paper. In addition, the new method is a general methodology which can be applied to any system, not limited to any concrete special systems. Therefore, the following fault trees omit each event's physical meaning.

\subsection{A Simple Fault Tree}

Taking Figure 1 for the first case, Figure 1 (a) is a fault tree, the equivalent Bayesian network mapped from the tree shown in Figure 1 (b), and the corresponding Jointree extracted from the Bayesian network shown in Figure 1 (c). All the bottom events' CPTs should be set to $\operatorname{Pr}\left(X_{i}=0\right)=1 / 2, \operatorname{Pr}\left(X_{i}=1\right)=1 / 2$ and the top event $\mathrm{T}$ should be set to $T=1$ in the Jointree according to the new method. After the inward and outward calculation, the results $\operatorname{Pr}\left(X_{1} \mid T=1\right), \operatorname{Pr}\left(X_{2} \mid T=1\right)$ and $\operatorname{Pr}\left(X_{3} \mid T=1\right)$ can be read from the cluster $\left\{X_{1}\right\},\left\{X_{2}\right\}$ and 
$\left\{X_{3}\right\}$ separately. Then according to the Eq. (3), all these three bottom events' structural importance degree can be computed. Apparently, inward and outward two times inference are needed here.The final results are shown in Table 1.

Table 1. Results of Case 1

\begin{tabular}{ccc}
\hline Bottom events & Old Bayesian network method & $\begin{array}{c}\text { New Bayesian network } \\
\text { method }\end{array}$ \\
\hline $\mathrm{X}_{1}$ & 0.75 & 0.75 \\
$\mathrm{X}_{2}$ & 0.25 & 0.25 \\
$\mathrm{X}_{3}$ & 0.25 & 0.25 \\
Number of inference & 3 times & 2 times \\
\hline
\end{tabular}

\subsection{A Fault Tree for a Power Distribution System}

Figure 2(a) is a fault tree for a power distribution system [12-13]. According to the steps for mapping fault trees into Bayesian networks, the achieved equivalent $\mathrm{BN}$ is shown in Figure 2. (b) and the Jointree mapped from the Bayesian network is shown in Figure 2 (c). It easily can be seen that all the bottom events' corresponding clusters can be found in the Jointree, separately. According to the new method, set every bottom event's CPT to $\operatorname{Pr}\left(X_{i}=0\right)=1 / 2, \quad \operatorname{Pr}\left(X_{i}=1\right)=1 / 2 \quad$ and $\mathrm{T}=1 . \quad$ Then the results $\operatorname{Pr}\left(X_{i}=0 \mid T=1\right), \operatorname{Pr}\left(X_{i}=1 \mid T=1\right)$ can be read from the Jointree after an inward and outward computation. Then all the bottom events' structural importance degree can be computed according to Eq. (3), results shown in Table 2, in which the results computed using the Eq.(2) (the old method) also provided.

From the results in Table 1 and Table 2, it can be seen that the same results can be computed by these two methods, which proves the correctness of the new method.

In case 1 , there are three bottom events, three times inference are performed using old method while two times inference are performed using the new algorithm; In case 2, there are eight bottom events, eight times inference are necessary using the old method while still two times inference are performed using the new algorithm. It can be concluded that as the number of bottom events $\mathrm{n}$ increases, the number of inference increases when using the old method. However, the number of inference is irrespective of $n$ when using the new method (only an inward and outward inference is necessary). 


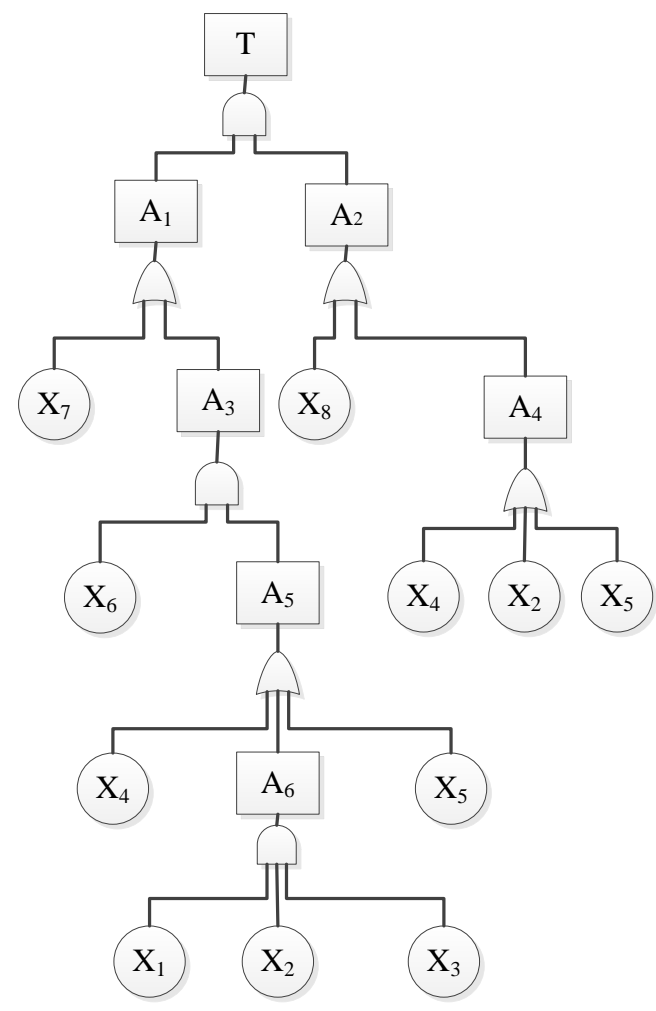

(a)

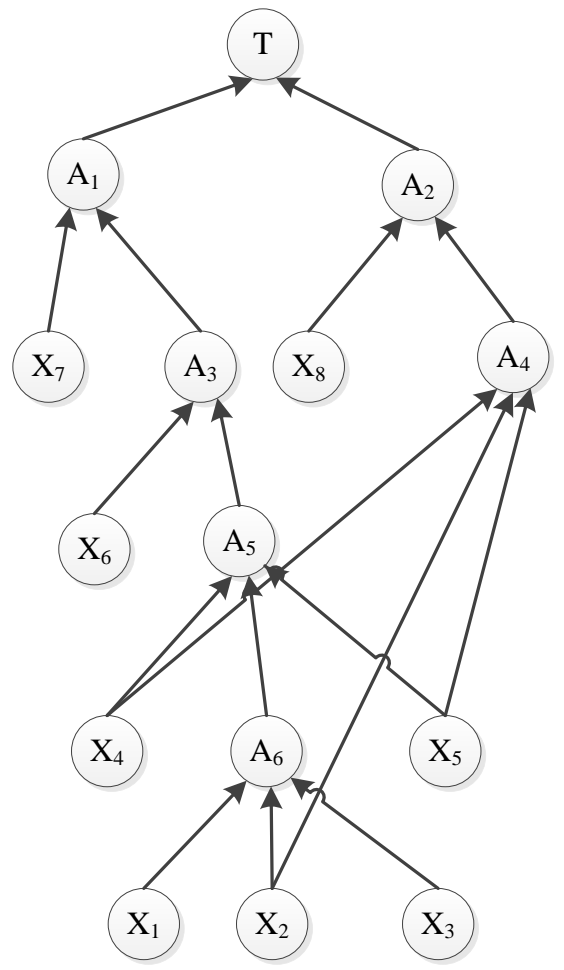

(b)

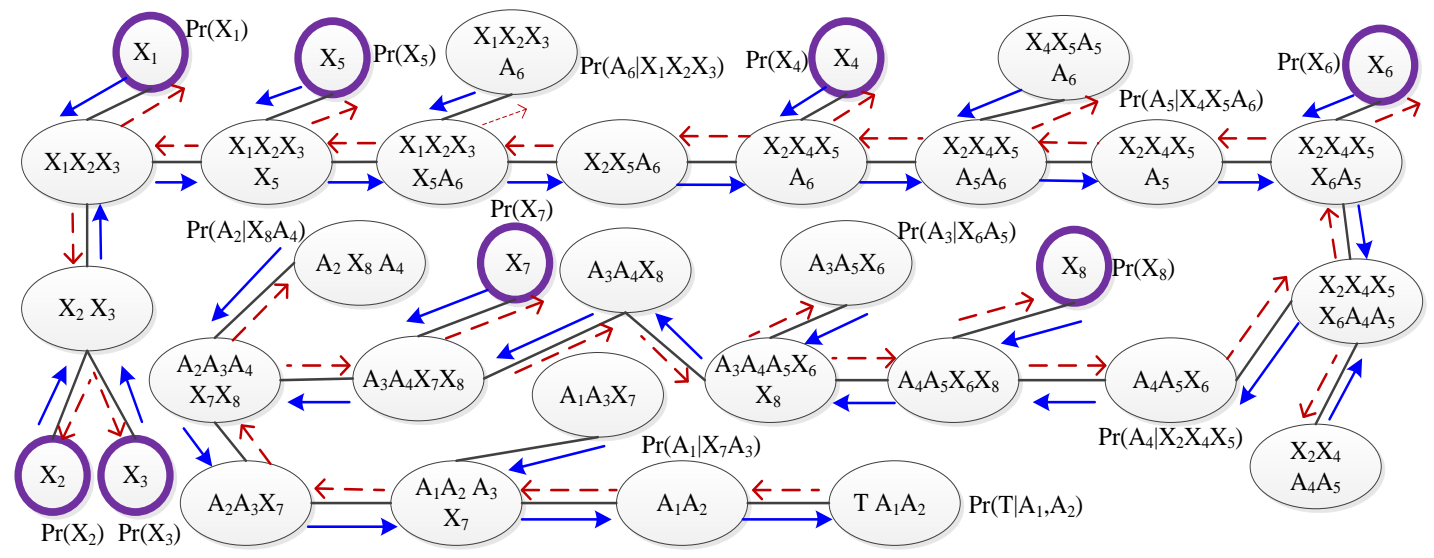

(c)

Figure 2. A Power Distribution System Described in Different Topologies

(a) A fault tree for a power distribution system; (b) Equivalent Bayesian network; (c) Jointree extracted from Bayesian network with each CPT assigned to their family clusters, taking the cluster $\left\{\mathrm{T}, \mathrm{A}_{1}, \mathrm{~A}_{2}\right\}$ as root, solid arrows indicate the direction of inward calculation and dotted arrows indicate the direction of outward calculation.

Table 2. Results of Case 2

\begin{tabular}{ccc}
\hline Bottom events $\quad$ Old Bayesian network method & $\begin{array}{c}\text { New Bayesian network } \\
\text { method }\end{array}$ \\
\hline
\end{tabular}




\begin{tabular}{ccc}
\hline $\mathrm{X}_{1}$ & 0.014649 & 0.014649 \\
$\mathrm{X}_{2}$ & 0.101563 & 0.101563 \\
$\mathrm{X}_{3}$ & 0.014649 & 0.014649 \\
$\mathrm{X}_{4}$ & 0.189456 & 0.189456 \\
$\mathrm{X}_{5}$ & 0.189453 & 0.189453 \\
$\mathrm{X}_{6}$ & 0.366211 & 0.366211 \\
$\mathrm{X}_{7}$ & 0.571289 & 0.571289 \\
$\mathrm{X}_{8}$ & 0.086914 & 0.086914 \\
Number of inference & 8 times & 2 times \\
\hline
\end{tabular}

\section{Conclusions}

On the basis of the existent exact methods for solving bottom event's structural importance degree including the traditional FTA method and the Bayesian network method raised by Zhou Zhongbao, combining with Bayesian network's inference characteristics, a new exact method for solving such an index together with the corresponding algorithm SID_Jointree is raised and proved to be correct in a mathematical way. Two cases are used to illustrate the new Bayesian network method in which a Jointree can be used effectively. Both practice and theory show that, as the bottom events' number $n$ increases, only two times computation is required using the new method instead of $\mathrm{n}$ times computation using the existent old method. As $\mathrm{n}$ increases, the new method is more effective, which is a feasible way to compute each bottom event's structural importance degree exactly and can be an useful assistant in analyzing a system's reliability.

\section{References}

[1] L. Portinale and A. Bobbio, "Bayesian networks for dependability analysis: an application to digital control reliability", In Proceedings of the fifteenth conference on uncertainty in artificial intelligence, Morgan Kaufmann Publishers Inc., (1999), July, pp. 551-558.

[2] H. Langseth and L. Portinale, "Bayesian networks in reliability", Reliability Engineering \& System Safety, vol. 92, no. 1, (2007), pp. 92-108.

[3] N. Khakzad, F. Khan and P. Amyotte, "Safety analysis in process facilities: Comparison of fault tree and Bayesian network approaches”, Reliability Engineering \& System Safety, vol. 96, no. 8, (2011), pp. 925-932.

[4] Y. Xiaowei, Q. Wenxue and X. Liyang, "A Method for System Reliabiltiy Assessment Based on Bayesian Networks [J]", Acta Aeronautica Et Astronautica Sinica, vol. 6, no. 013, (in Chinese), (2008).

[5] G. Weidl, A. L. Madsen and S. Israelson, "Applications of object-oriented Bayesian networks for condition monitoring, root cause analysis and decision support on operation of complex continuous processes", Computers \& chemical engineering, vol. 29, no. 9, (2005), pp. 1996-2009.

[6] Z. Zhongbao, D. Doudou and Z. Jinglun, "Application of Bayesian Networks in Reliability Analysis [J]", Systems Engineering-Theory \& Practice, vol. 6, (2006), pp. 95-100 (in Chinese).

[7] A. Darwiche, "Modeling and reasoning with Bayesian networks", New York: Cambridge University Press, (2012).

[8] Z. Lianwen and G. Haipeng, "An Introduction to Bayesian Networks", Beijing: Science Press (in Chinese), (2006).

[9] P. P. Shenoy, "Binary join trees for computing marginals in the Shenoy-Shafer architecture", International Journal of approximate reasoning, vol. 17, no. 2, (1997), pp. 239-263.

[10] K. Lingasubramanian, S. M. Alam and S. Bhanja, "Maximum error modeling for fault-tolerant computation using maximum a posteriori (MAP) hypothesis", Microelectronics Reliability, vol. 51, no. 2, (2011), pp. 485501.

[11] D. Qiong, "Safety system engineering", Xi'an: Northwest Industrial University press (in Chinese), (2009).

[12] S. Mahadevan, R. Zhang and N. Smith, "Bayesian networks for system reliability reassessment", Structural Safety, vol. 23, no. 3, (2001), pp. 231-251. 
[13] E. Castillo, C. Solares and P. Gómez, "Estimating extreme probabilities using tail simulated data", International Journal of Approximate Reasoning, vol. 17, no. 2, (1997), pp. 163-189.

\section{Authors}

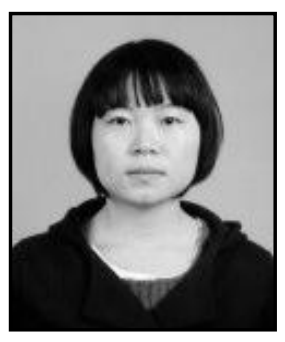

Wang Yao, She received her B.Eng. in Aircraft Design and Engineering (2011) and is currently doing a doctorate in Aircraft Design at School of Aeronautics, Northwestern Polytechnical University as a full-time student (Since 2011). Her current research interests include the Aircraft system reliability assessment and fault diagnosis.

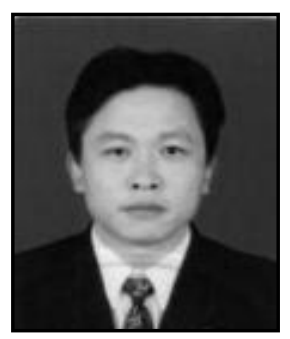

Sun Qin, He received his PhD in Aircraft Design from Northwestern Polytechnical University. Now he is full professor of Aircraft Design at School of Aeronautics, Northwestern Polytechnical University. He is the chairman of the structure design and strength of China aviation committee. He has published a total of more than 100 academic papers (10/SCI, 50/EI, 20/ISTP). His current research interests include the aircraft structural design, aircraft system design and reliability evaluation. 
International Journal of $u$ - and e- Service, Science and Technology Vol.8, No.1 (2015) 